\title{
A Transient UWB Antenna Array Used with Complex Impedance Surfaces
}

\author{
A. Godard, ${ }^{1}$ L. Desrumaux, ${ }^{1}$ V. Bertrand, ${ }^{2}$ J. Andrieu, ${ }^{1}$ M. Lalande, ${ }^{1}$ B. Jecko, ${ }^{3}$ \\ V. Couderc, ${ }^{4}$ M. Brishoual, ${ }^{5}$ and R. Guillerey ${ }^{6}$ \\ ${ }^{1}$ XLIM/OSA, IUT GEII, 7 rue Jules Vallès, 19100 Brive, France \\ ${ }^{2}$ CISTEME, ESTER, BP 6913, 87069 Limoges Cedex, France \\ ${ }^{3}$ XLIM/OSA, Université de Limoges, 23 Avenue Albert Thomas, 87060 Limoges Cedex, France \\ ${ }^{4}$ XLIM/PHOTONIQUE, Université de Limoges, 123 Avenue Albert Thomas, 87060 Limoges Cedex, France \\ ${ }^{5}$ DGA IP/CGN/DE, 7 rue des Mathurins, 92220 Bagneux, France \\ ${ }^{6}$ CELAR CGN/MSE, BP 7419, 37174 Bruz Cedex, France
}

Correspondence should be addressed to A. Godard, adriengodard@gmail.com

Received 2 October 2009; Revised 14 February 2010; Accepted 9 April 2010

Academic Editor: Karu Esselle

Copyright ( $) 2010$ A. Godard et al. This is an open access article distributed under the Creative Commons Attribution License, which permits unrestricted use, distribution, and reproduction in any medium, provided the original work is properly cited.

\begin{abstract}
The conception of a novel Ultra-Wideband (UWB) antenna array, designed especially for transient radar applications through the frequency band $(300 \mathrm{MHz}-3 \mathrm{GHz})$, is proposed in this paper. For these applications, the elementary antenna must be compact and nondispersive, and the array must be able to steer in two dimensions. The geometry of the elementary antenna and its radiation characteristics are presented. The array beam steering is analyzed and a technique making the increase of the transient front-toback ratio possible is described.
\end{abstract}

\section{Introduction}

In the recent years, investigations directed to the realization of Ultra-Wideband (UWB) systems that radiate transient waveforms and exhibit operating bandwidths of over one decade are made intensively in many countries. Such systems radiate impulses with rise times around 100 ps and duration of few nanoseconds. Such radiated waveforms make them interesting for many applications such as transient radar cross section (RCS) measurements [1] or UWB synthetic aperture radar (SAR) systems [2]. The main interest of such systems is that the radar resolution is proportional with the radiated bandwidth. Contrary to narrowband systems, a second advantage is the capacity of identifying stealth targets.

This paper describes a novel antenna array appearing in the $\mathrm{N}$ generators/ $\mathrm{N}$ antennas configuration, because it presents the advantage of increasing the radiation power on one hand and offering the agility to the array on the other hand. Moreover, this architecture permits a working continuity even if an elementary source is defective. With the frequency spectrum $[300 \mathrm{MHz}-3 \mathrm{GHz}]$, this kind of system permits to detect hidden objects (wall, foliage, or ground penetration, etc.).

A major difficulty of limiting the synchronization of the radiation source jitters yet exists. A solution which consists in using impulse optoelectronic devices permits to bypass this difficulty, with the generation of ultra short electrical waveforms with a small jitter (2 ps typically) [3].

Section 2 presents the elementary antenna and its radiation characteristics. Then, the array beam steering is analyzed in Section 3, and a technique involving a diminution of the back radiations and the amount of radiations in the transverse plane is presented in Section 4. Indeed, the increase of the transient front-to-back ratio is essential to limit fratricidal effects.

\section{The Elementary Antenna}

The elementary antenna is inspired by a travelling wave antenna, the Valentine antenna [4], because it has good linearity properties. Moreover, the antenna developed by 


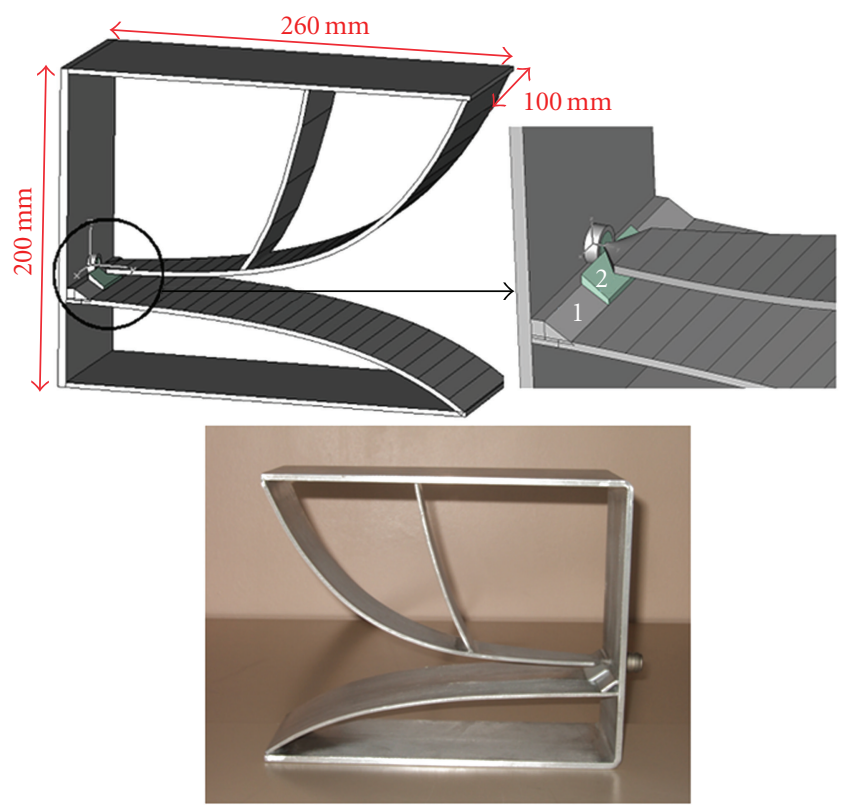

Figure 1: The elementary antenna (CAD model and realized geometry).

Koshelev et al. [5] is another good starting point in order to reduce its dimensions. Figure 1 presents the elementary antenna and its adequate transition: piece 1 is made of metal while piece 2 is made of PTFE and permits a high-voltage resistance. This antenna has been designed with the CST Microwaves Studio software and can support a transient voltage of $10 \mathrm{kV}$, with a pulse width of 2 nanoseonds and a repetition frequency equal to $1 \mathrm{kHz}$.

Figure 2 shows how important this adequate transition is. Indeed, with this optimized transition, the antenna is well matched $\left(S_{11}\right.$ parameter lower than $\left.-10 \mathrm{~dB}\right)$ over the desired wide frequency band [ $300 \mathrm{MHz}-3 \mathrm{GHz}$ ].

The maximum wavelength of the spectrum that has to be covered is $\lambda_{300 \mathrm{MHz}}=1 \mathrm{~m}$. Thus, the general size of the antenna is

(i) $10 \mathrm{~cm}=\lambda_{300 \mathrm{MHz}} / 10$ for the width,

(ii) $20 \mathrm{~cm}=\lambda_{300 \mathrm{MHz}} / 5$ for the height,

(iii) $26 \mathrm{~cm}=\lambda_{300 \mathrm{MHz}} / 3,85$ for the length.

These dimensions, which can be considered as small, allow the conception of a $2 \mathrm{D}$ array. Figure 3 presents the gain of this antenna, both in simulation (with the CST microwaves Studio software) and in measurement.

The gain increases with the frequency, from $0 \mathrm{dBi}$ at $300 \mathrm{MHz}$ to $10 \mathrm{dBi}$ at $3 \mathrm{GHz}$.

Transient UWB antennas are also characterized by their transient front-to-back ratio. The one of this antenna is $19,6 \mathrm{~dB}$, which will involve a high transient front-to-back ratio as far as the array is concerned.

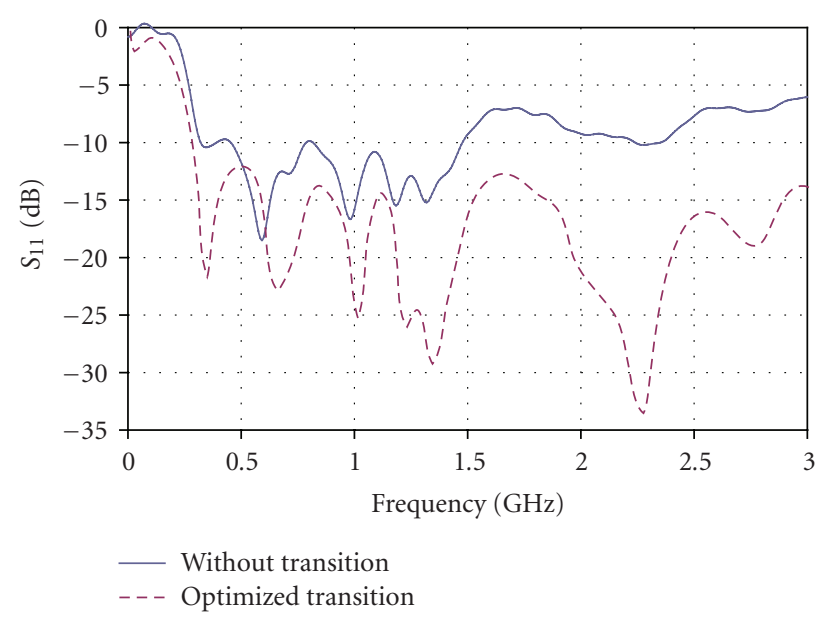

Figure 2: Reflection coefficient.

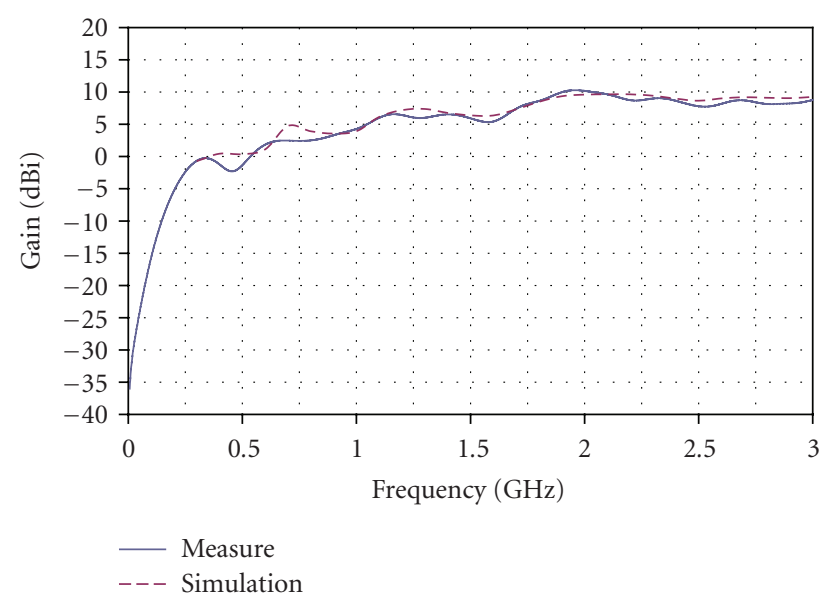

FIGURE 3: Gain of the elementary antenna.

\section{Antenna Array}

The antenna presented in Section 2 is now integrated in a 4 $\times 3$ array (Figure 4 ).

3.1. Design of the Array. Each antenna is firmly attached to the others along the vertical axis to reduce the length between each feeding port, which involves a reduction of the side lobes level in the E-plane. However, it is not the case in the horizontal axis. The best compromise between a low coupling (which is high when the antennas are in contact) and a small side lobes level (which is low when the antennas are in contact) is obtained with a distance between antennas equal to $20 \mathrm{~mm}$.

The behavior of UWB systems can be predicted by means of the transfer function of the antenna in transmission $h_{T}$, defined in the following expression:

$$
h_{T}(t)=\mathrm{FT}^{-1}\left[\sqrt{\frac{4 \pi Z_{C}}{Z_{0}}} \times \frac{E(d, f)}{V_{g}(f)} \times d \times e^{j 2 \pi f d}\right],
$$

where $Z_{C}$ is the antenna characteristic impedance, $Z_{0}$ is the intrinsic impedance of free space $(120 \pi), E$ is the radiated 


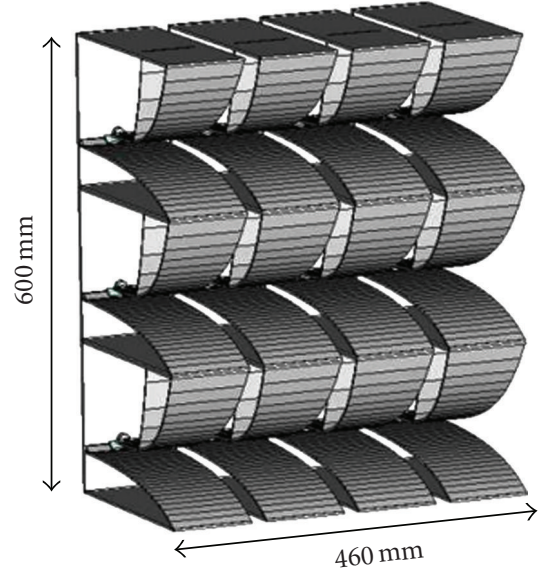

Figure 4: The antenna array.

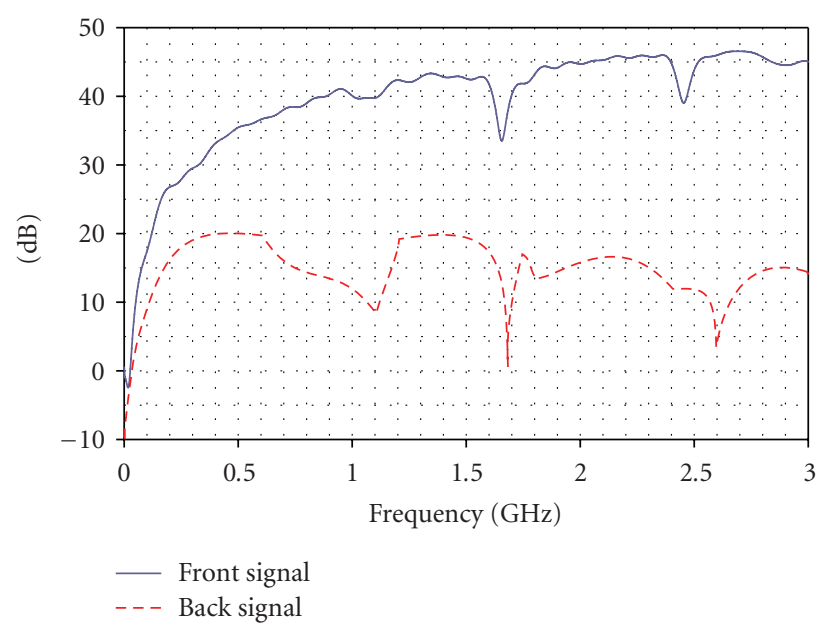

FIgURE 5: Radiated signals in the frequency domain. electric farfield (in the frequency domain), $V_{g}$ is the signal applied to the antenna (in the frequency domain), $d$ is the distance where the radiated field is evaluated, $f$ is the considered frequency, and $\mathrm{FT}^{-1}$ is the inverse Fourier Transform.

As mentioned in the introduction, the array is fed by 12 independent generators. Thus, the beam forming is obtained by applying different time delays on the antennas, and the field radiated by one antenna in any direction can be defined with the following expression:

$$
\begin{aligned}
& e_{T}(t, d, \theta, \psi) \\
& \quad=\frac{1}{d} \sqrt{\frac{Z_{0}}{4 \pi Z_{C}}}\left[h_{T}(t, \theta, \psi) \otimes v_{g}(t) \otimes \delta\left(t-\frac{d}{c}-\tau\right)\right],
\end{aligned}
$$

where $\otimes$ is the convolution operator, $v_{g}$ is the transient signal applied to the antenna, $\delta$ is the Dirac function, $c$ is the light velocity in vacuum, and $\tau$ is the time delay applied to each antenna.

The time delay $\tau$ applied to each antenna is characterized by the Dirac function. One delay per beam steering has been defined: $\tau_{h}$ for the horizontal one and $\tau_{v}$ for the vertical one:

$$
\tau_{h}=\frac{\left(N_{h}-1\right) X_{h} \sin (\theta)}{c}, \quad \tau_{v}=\frac{\left(N_{v}-1\right) X_{v} \sin (\psi)}{c},
$$

where $N_{h}$ and $N_{v}$ are the antennas numbers, respectively, in row and in column, $X_{h}$ and $X_{v}$ are the distances between two feeding ports, respectively, in the horizontal and the vertical plane, and $\theta$ and $\psi$ define the direction of the beam-steering, respectively, in the horizontal and the vertical plane.

As a result, the total transient-radiated field of the antenna array is the coherent sum of the different single elements:

$$
\begin{aligned}
& e_{\text {tot }}(t, d, \theta, \psi) \\
& \quad=\frac{1}{d} \sqrt{\frac{Z_{0}}{4 \pi Z_{C}}} \sum_{i=1}^{N}\left[h_{T}(t, \theta, \psi) \otimes v_{g}(t) \otimes \delta_{i}\left(t-\frac{d}{c}-\tau_{i}\right)\right],
\end{aligned}
$$

where $N$ is the antenna number, and the delay $\tau_{i}$ is the sum of $\tau_{h}$ and $\tau_{v}$ (each $i$ th antenna corresponds to a couple $\left(N_{h}\right.$; $\left.N_{v}\right)$ ).

3.2. Radiation Characteristics. This part presents the radiation characteristics of the $4 \times 3$ array. The following results have been obtained via the CST Microwaves Studio software, which takes into account every problem in relation with coupling effects or mismatch. The signal delivered by the generator is a null mean value pulse whose bandwidth is corresponding to $[300 \mathrm{MHz}-3 \mathrm{GHz}]$.

Figure 5 presents the radiated signals both in front of the array and at the back of it, in the frequency domain. This figure shows how high the front-to-back ratio is. Moreover, the transient front-to-back ratio of the array is $28,3 \mathrm{~dB}$, which is a great increase in comparison with the one of the elementary antenna. This ratio is the reference for further analysis.

The graph which represents the transient electric field as function of time and angle is a suitable UWB radiation source descriptor [6]. Figures 6 and 7 represent, respectively, in the $\mathrm{H}$-plane and in the E-plane, this type of characteristic when all the antennas are simultaneously fed and the delay between feeding allows a steering angle of $12^{\circ}$.

Contrary to harmonic radars, for which the criterion permitting to evaluate the beam steering capabilities of an array is the side lobes level, transient arrays can be characterized by the time dispersion and the decreasing of the peak level of the radiated field. The higher the steering angle is, the lower is the maximum peak level and the higher is the dispersion, as shown in Figure 8. This figure represents the transient-radiated field in the $\mathrm{H}$-plane in two configurations: without steering and with a $15^{\circ}$ steering angle.

Concerning the level, the reduction can be approximated, in this case, to $1 \%$ per degree. Moreover, concerning the time duration, the increase is about $2,5 \mathrm{ps}$ per degree. If the peak level of the radiated field is not high enough, the analyzed distance will be too short. If the radiated field is too 

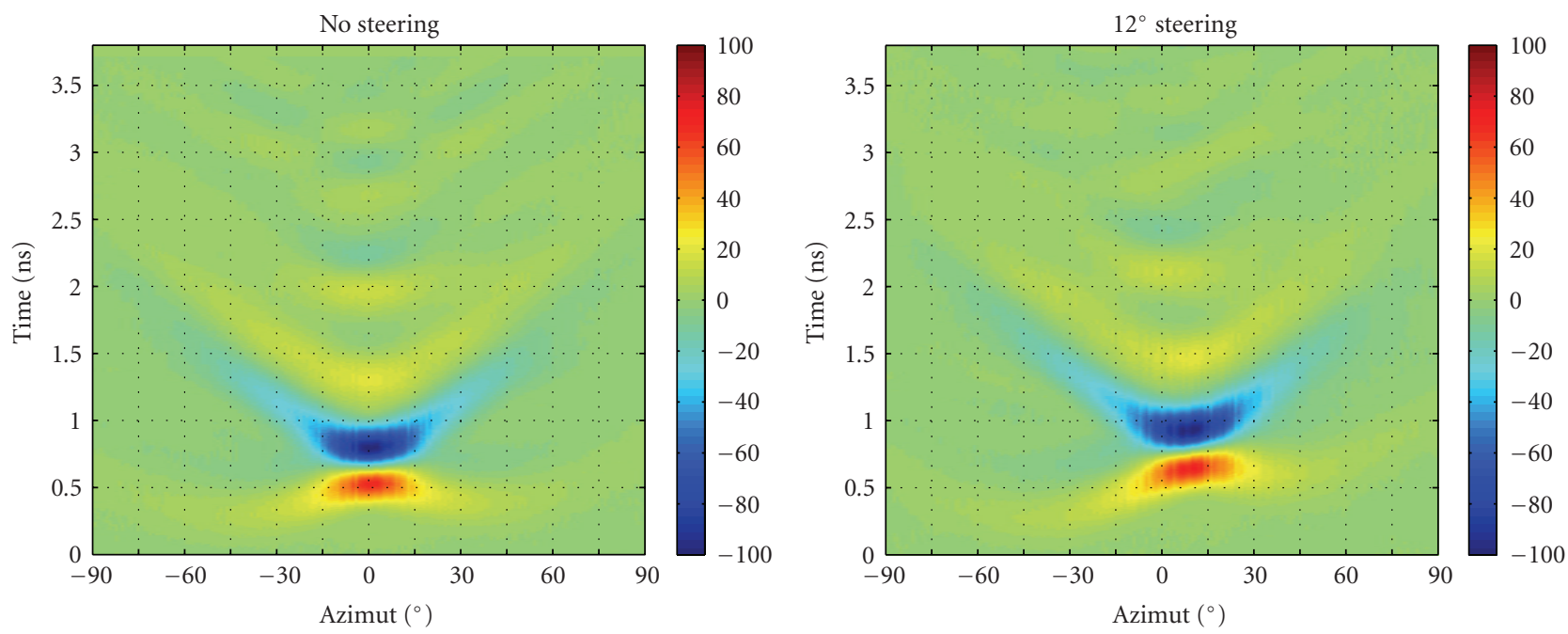

FIgURE 6: Transient radiation patterns in the H-plane.
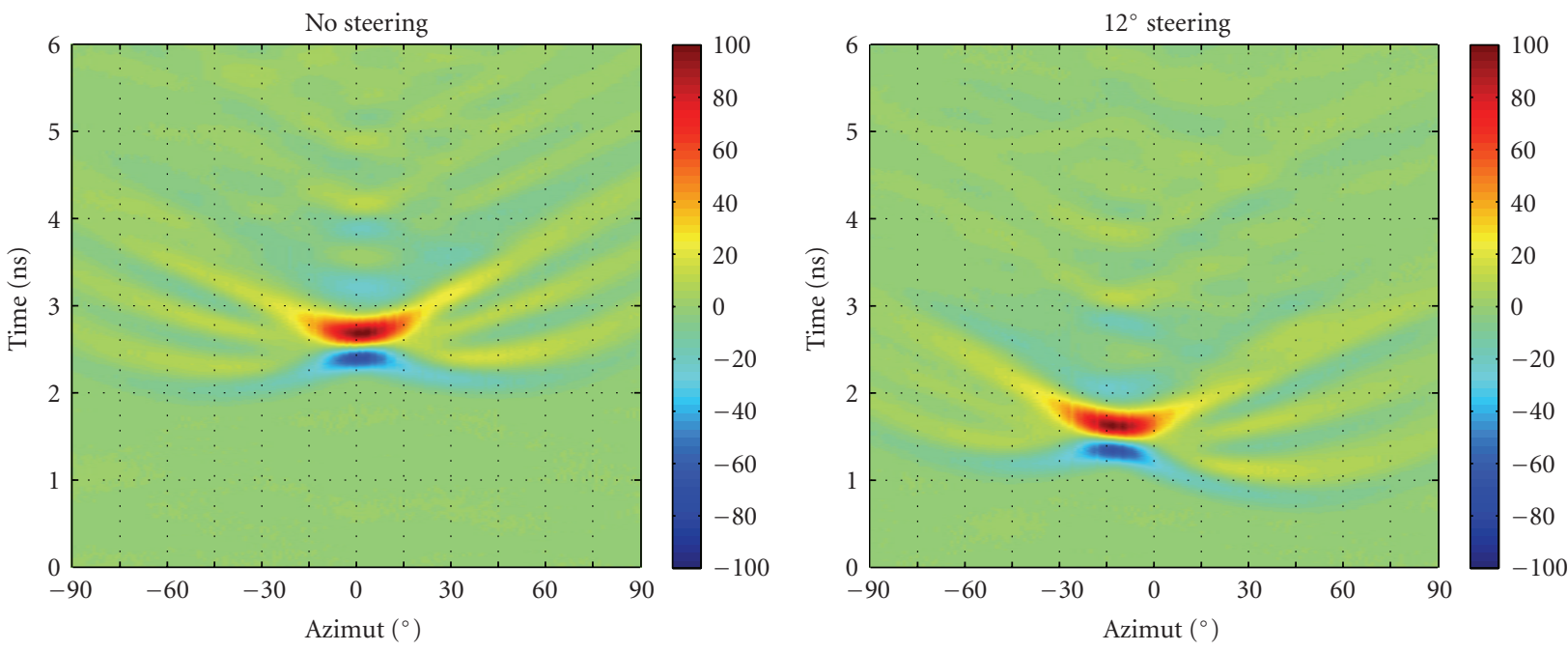

FIgURE 7: Transient radiation patterns in the E-plane.

dispersive, the radar imaging will become less accurate. Thus, the acceptable transient steering angle value depends on the application.

\section{Back Radiation Optimization}

The weakness of UWB antenna arrays composed of travelling wave antennas is the back radiations. In order to reduce these back radiations, the work leads to the behavior of the global system rather than the behavior of the elementary antenna. Moreover, a technique consisting in decreasing the back radiations without using low-frequency absorbers, which are much too big to be used in embedded systems, is necessary.

This part deals with the use of periodic structures on UWB arrays. These periodic structures are, by their design, harmonic structures. The impact of corrugations, which are Frequency Selective Surfaces (FSS), on the transient-radiated field is presented. Those surfaces were first used as reflectors or in corrugated horn [7-9].

4.1. Definition of a Complex Surface. A complex surface can be characterized by a complex impedance $Z_{S}=R_{S}+j X_{S}$, as shown in Figure 9.

Along the $x$-axis, a TM wave can be considered, with a complex propagation constant $\beta=\beta^{\prime}-j \beta^{\prime \prime}$ and a complex transverse wave number $h=h^{\prime}+j h^{\prime \prime}$ :

$$
H_{Z}=A \exp (j h y-j \beta x) .
$$




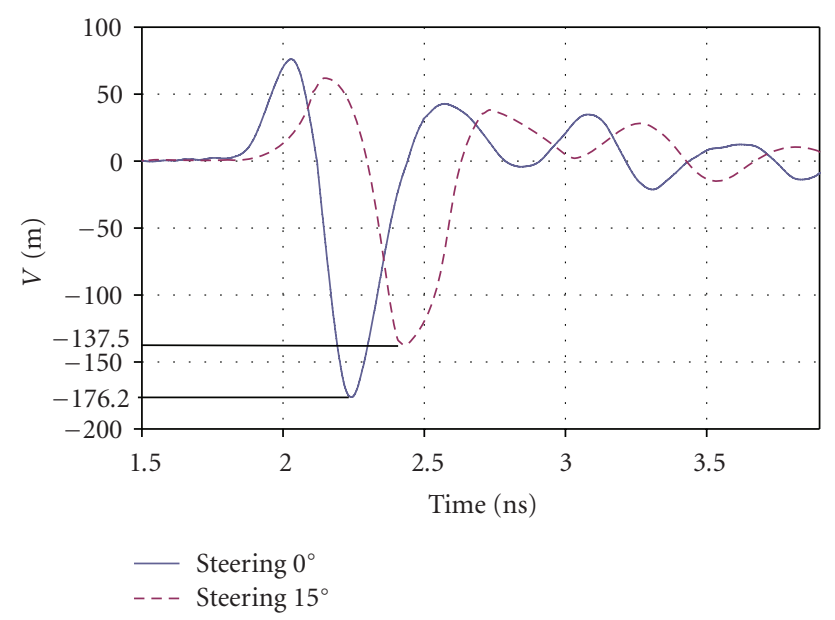

Figure 8: Beam steering effects on the transient-radiated field.

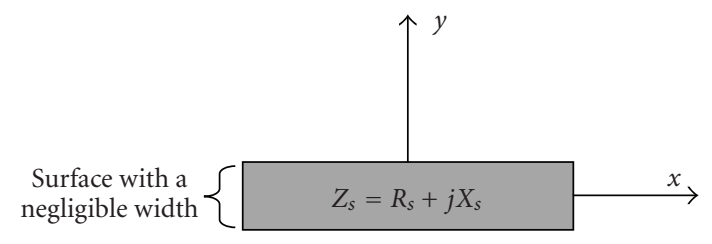

FIgURE 9: Complex surface.

In accordance with the Maxwell-Ampère law, the electromagnetic field can be calculated. $E$ is the electric field and $H$ is the magnetic field:

$$
\begin{aligned}
r \overrightarrow{o t}(\vec{H})= & \varepsilon_{0} \frac{\partial \vec{E}}{\partial t} \\
\operatorname{rot}(\vec{H})= & \left(\frac{\partial H z}{\partial y}-\frac{\partial H y}{\partial z}\right) \vec{x}+\left(\frac{\partial H x}{\partial z}-\frac{\partial H z}{\partial x}\right) \vec{y} \\
& +\left(\frac{\partial H y}{\partial x}-\frac{\partial H x}{\partial y}\right) \vec{z} .
\end{aligned}
$$

For a TM wave along the $x$-axis, only the $H z$ component exists; therefore $E x$ and $E y$ can be determined:

$$
j w \varepsilon_{0} E x=\frac{\partial H z}{\partial y}, \quad j w \varepsilon_{0} E y=-\frac{\partial H z}{\partial x} .
$$

Then, with $k_{0}^{2}=h^{2}+\beta^{2}$, the wave impedance $Z_{l}$ is

$$
Z_{l}=\frac{E x}{H z}=\frac{h}{w \varepsilon_{0}}=\frac{h}{k_{0}} Z_{0}
$$

To verify boundary limits on the surface $\left(y=0, Z_{l}=Z_{S}\right)$,

$$
\begin{gathered}
h=h^{\prime}+j h^{\prime \prime}=\frac{k_{0} Z_{S}}{Z_{0}}=\frac{k_{0} R_{S}}{Z_{0}}+j \frac{k_{0} X_{S}}{Z_{0}}, \\
\beta=\beta^{\prime}-j \beta^{\prime \prime}=\sqrt{k_{0}^{2}-h^{2}} .
\end{gathered}
$$

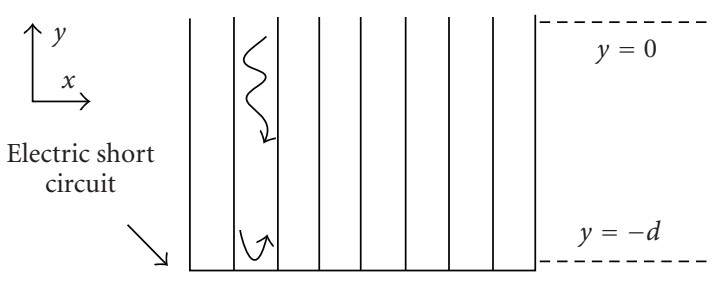

Figure 10: Corrugated surface.

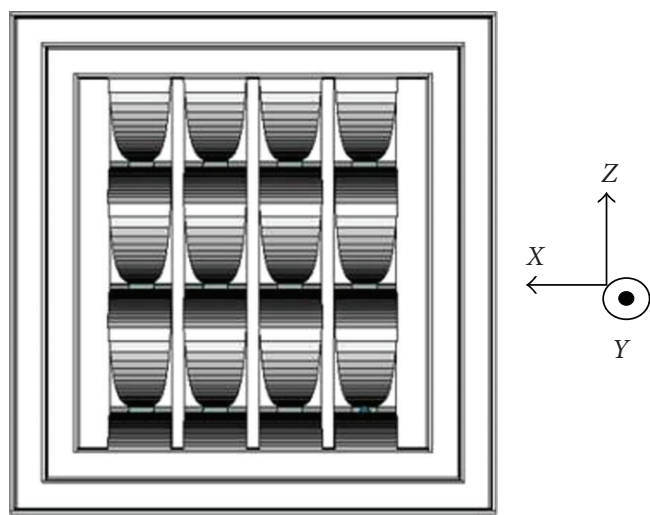

Figure 11: UWB antenna array with corrugations.

As a result, the surface wave can be written as

$$
H_{Z}=A \exp \left(j \frac{k_{0} Z_{S}}{Z_{0}} y-j k_{0} \sqrt{1-\frac{Z_{S}^{2}}{Z_{0}^{2}} x}\right) .
$$

This formulation is generic and may be used with different complex surfaces. In this case, the chosen surface is a corrugated one. Section 4.2 is dedicated to the use of corrugated surfaces on the UWB array presented in Section 3.

4.2. Corrugated Surface. As shown in Figure 10, the corrugations are assimilated to a transmitting line short circuited on its end $[10,11]$. In this case, the distance $d$ is equal to $260 \mathrm{~mm}$, which corresponds to the length of the elementary antenna.

The input impedance on the surface corresponding to $y=0$ is calculated with the line theory and the result is

$$
Z_{S}=j X_{S}=j Z_{C} \tan \left(\frac{2 \pi}{\lambda} d\right)
$$

where $Z_{C}$ is the characteristic impedance of the corrugation, assimilated to an equivalent transmission line.

Thus, the parameters $h$ and $\beta$ become

$$
\begin{aligned}
& h=\frac{k_{0}}{Z_{0}} Z_{S}=j k_{0} \frac{Z_{C}}{Z_{0}} \tan \left(\frac{2 \pi}{\lambda} d\right), \\
& \beta=\sqrt{k_{0}^{2}-h^{2}}=k_{0} \sqrt{1-\left(\frac{Z_{S}}{Z_{0}}\right)^{2}} .
\end{aligned}
$$



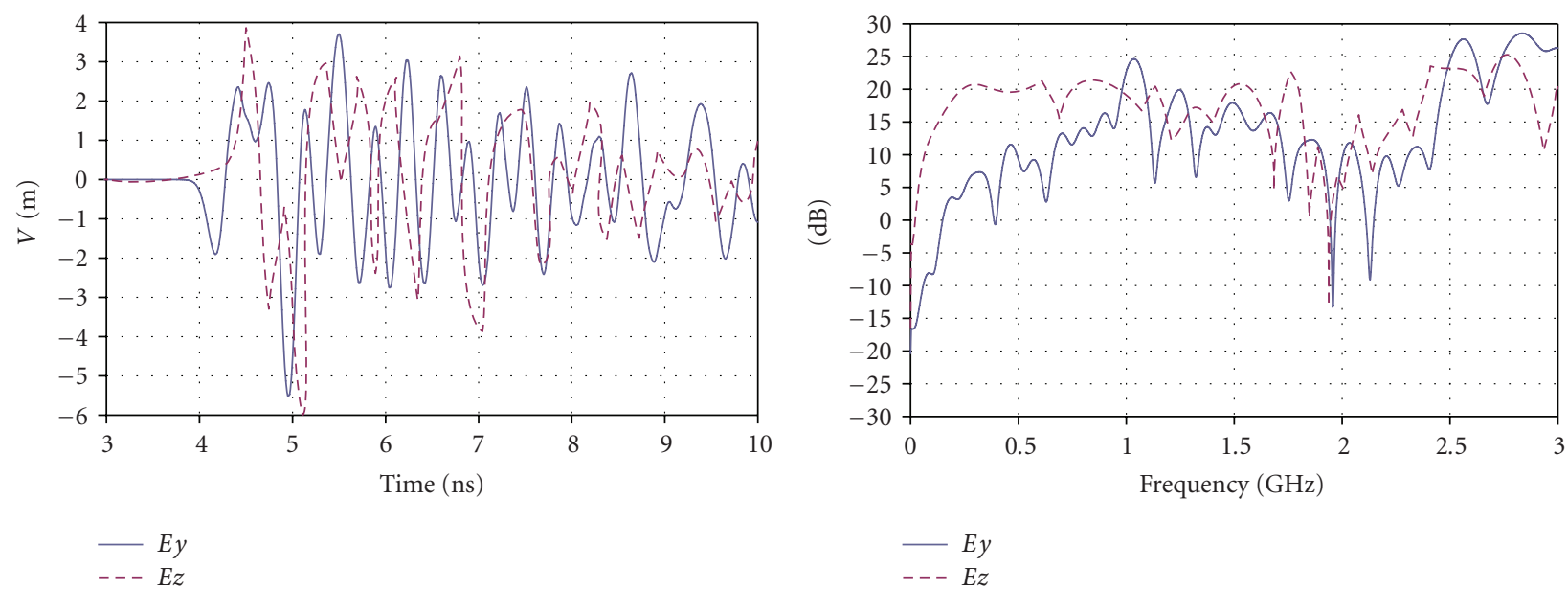

FIGURE 12: Radiated fields along the $x$-axis without corrugations.
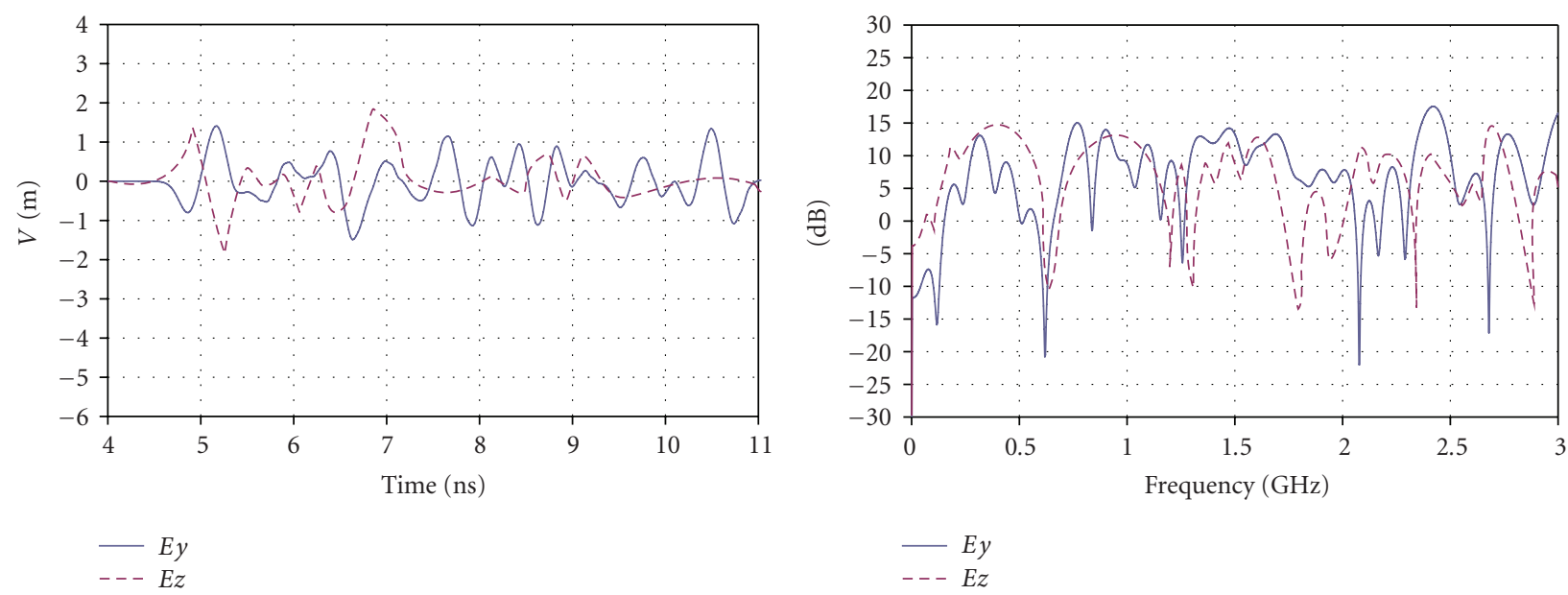

FIgURE 13: Radiated fields along the $x$-axis with corrugations.
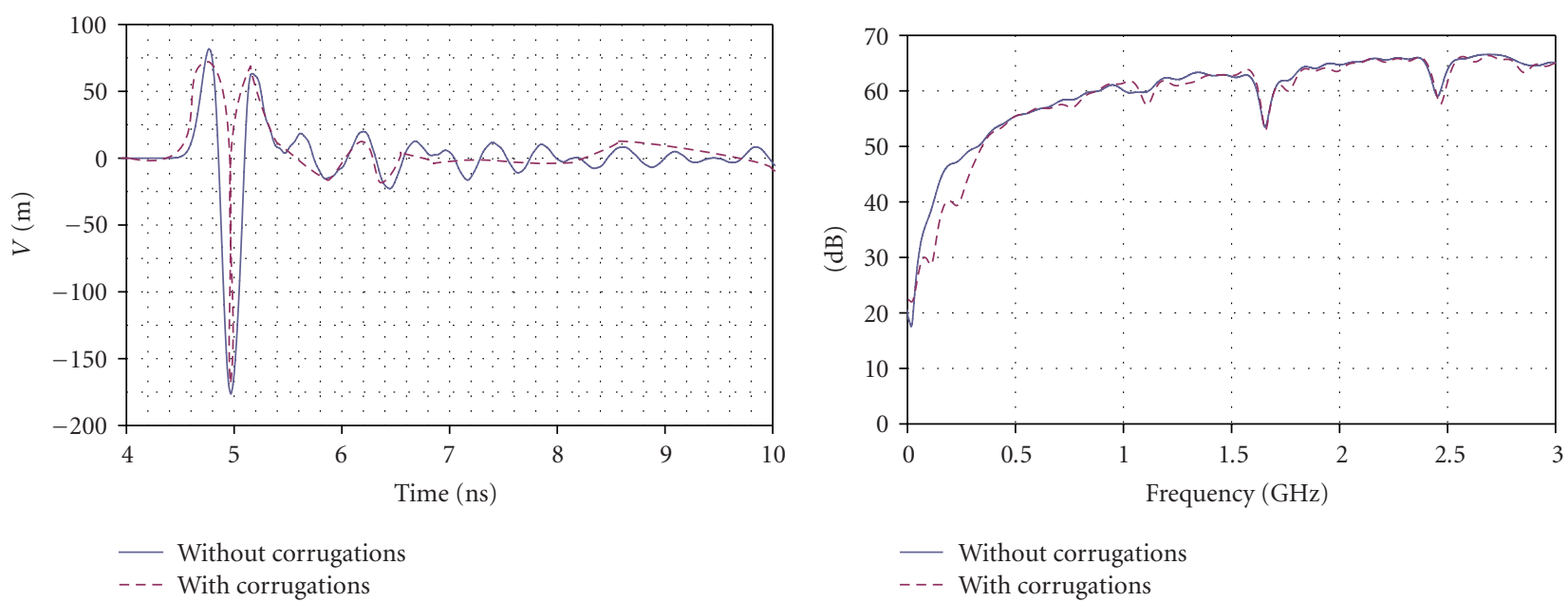

Figure 14: Front signal $(E z)$ with and without corrugations. 

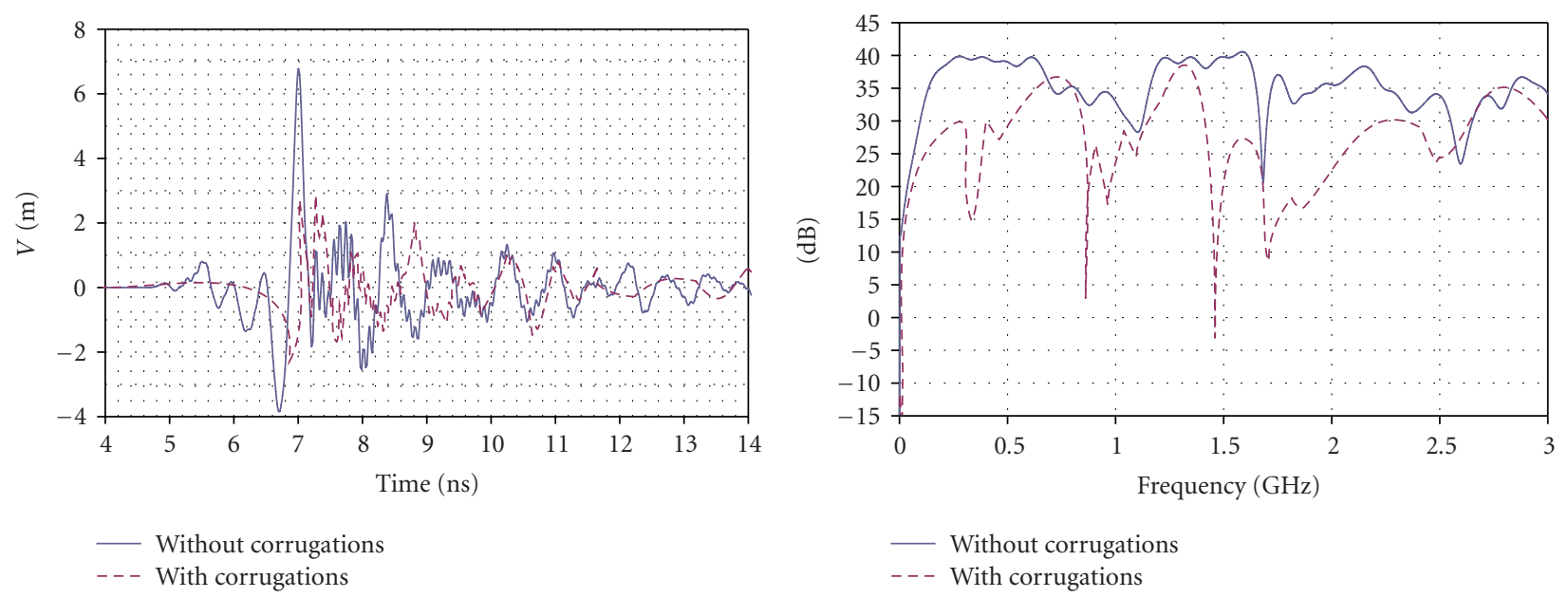

FIGURE 15: Back signal $(E z)$ with and without corrugations.

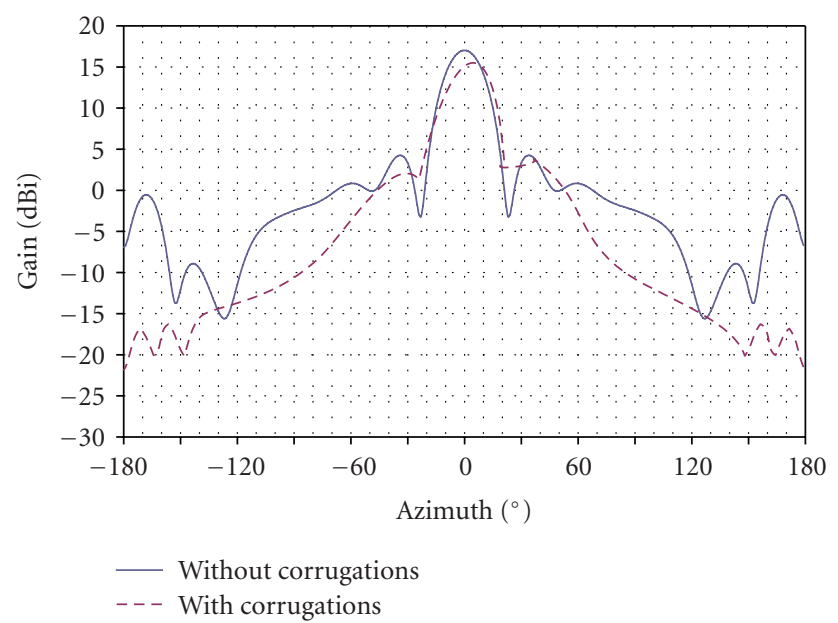

FIgURE 16: Gain of the array at 1,44 GHz in the H-plane.

Introducing these parameters in (10), $\mathrm{Hz}$ become

$$
\begin{aligned}
H_{Z}=A \exp ( & -\frac{Z_{C}}{Z_{0}} k_{0} \tan \left(\frac{2 \pi d}{\lambda}\right) y \\
& -j k_{0} \sqrt{\left.1-\left(\frac{Z_{C}}{Z_{0}}\right)^{2} \tan \left(\frac{2 \pi d}{\lambda}\right)^{2} x\right)},
\end{aligned}
$$

and the attenuation is due to $A \exp \left(-\left(Z_{C} / Z_{0}\right) k_{0} \tan (2 \pi d /\right.$ ג) $y)$.

For a distance $d$ equal to $\lambda / 4+p(\lambda / 2), p \in N$, the frequencies corresponding to $\lambda$ are forbidden. Therefore, the transient signal is disturbed and reduced at these frequencies.

4.3. Corrugations and UWB Array. As shown in Figure 11, the corrugations surround the array in all directions. Their depth is the same and is equal to $260 \mathrm{~mm}$.

Figures 12 and 13 present, respectively, without and with corrugations, the radiated fields along the $x$-axis, which is the direction where the corrugations have a great impact.
Figure 13 shows that the corrugations work perfectly on both polarization $E y$ and $E z$. Indeed, these components are reduced with a factor higher than $50 \%$. Along the $z$-axis, the transient signal is not reduced as well as along the $x$ axis. Indeed, the corrugations are not well oriented for this polarization.

Figures 14 and 15 show the influence of the corrugations, which are Frequency Selective Surfaces (FSS), on the front and back signals, both in the time and in the frequency domains. The dotted line (Figure 15) shows three frequencies where the back signal is reduced. All these frequencies have their corresponding wavelength that is multiple of $4 \mathrm{~d}$ : $\mathrm{fc} 1=$ $288 \mathrm{MHz}, \mathrm{fc} 2=865 \mathrm{MHz}$, and fc3 $=1.44 \mathrm{GHz}$.

The transient front-to-back ratio has changed due to these forbidden frequencies; it is now equal to $35 \mathrm{~dB}$.

The front field peak level has been slightly reduced, and the back field peak level has been reduced much more, which involves an increase of $7 \mathrm{~dB}$ on the transient front-to-back ratio.

Moreover, the energy not dissipated at the back of the array, due to the use of corrugations, goes in other directions. For example, Figure 16 represents the gain of the array with and without corrugations at $1,44 \mathrm{GHz}$ (which is one of the three forbidden frequencies), in the H-plane.

This figure shows that the gain of the array with corrugations is higher than the gain of the array without corrugations in three directions. Indeed, there is a difference equal to $0,3 \mathrm{~dB}$ at the azimuth $0^{\circ}$ and $4,29 \mathrm{~dB}$ at $+/-24^{\circ}$.

\section{Conclusion}

The miniature antenna presented in this paper is suitable for transient UWB radar applications in the frequency band [300 MHz-3 GHz]. The chosen configuration is composed of $\mathrm{N}$ generators and $\mathrm{N}$ antennas because it offers the agility to the array and permits a working continuity even if an elementary source is defective. Each antenna is fed with an ultra short electrical waveform generated by an impulse optoelectronic device. This solution permits to limit the jitter between the sources. 
The $4 \times 3$ array presented in this paper makes possible the realization of a transient beam steering in two dimensions. Moreover, the method consisting in using corrugations all around the array offers great possibilities to reduce back radiations level with a low cost of space. Indeed, the corrugations disturb the back radiations at some frequencies and behave the same way whatever the steering angle is.

\section{Acknowledgment}

This work is supported by the French armament procurement agency DGA, DGA/MRIS (Mission for Research and Scientific Innovation).

\section{References}

[1] I. I. Immoreev and J. D. Taylor, "Optimal short pulse ultrawideband radar signal detection," in Ultra-Wideband ShortPulse Electromagnetics 5, P. D. Smith and S. R. Cloude, Eds., pp. 207-214, Kluwer Academic/Plenium Publishers, Dordrecht, The Netherlands, 2002.

[2] M. A. Ressler, "The army research laboratory ultra wideband boomSAR," in Proceedings of International Geoscience and Remote Sensing Symposium (IGARSS '96), vol. 3, pp. 18861888, Lincoln, Neb, USA, May 1996.

[3] M. Lalande, J. C. Diot, S. Vauchamp, et al., "An ultra wideband impulse optoelectronic radar: RUGBI," Progress in Electromagnetics Research B, no. 11, pp. 205-222, 2009.

[4] J. C. Diot, P. Delmote, J. Andrieu, et al., "A novel antenna for transient applications in the frequency band $300 \mathrm{MHz}$ 3 GHz: the Valentine antenna," IEEE Antennas and Propagation Networks, vol. 55, no. 3, pp. 987-990, 2007.

[5] V. I. Koshelev, V. P. Gubanov, A. M. Efremov, et al., "Highpower ultra-wide band radiation source with multielement array antenna," in Proceedings of the 13th International Symposium on High Current Electronics, Tomsk, Russia, July 2004.

[6] G. R. Salo and J. S. Gwynne, "UWB antenna characterization and optimization methodologies," in Ultra-Wideband ShortPulse Electromagnetics 6, E. L. Mokole, M. Kragalott, and K. R. Gerlach, Eds., pp. 329-336, Kluwer Academic/Plenium Publishers, Dordrecht, The Netherlands, 2003.

[7] B. A. Auld, J. J. Gagnepain, and M. Tan, "Horizontal shear surface waves on corrugated surfaces," Electronics Letters, vol. 12, no. 24, 1976.

[8] R. E. Collin, Field Theory of Guided Waves, IEEE Press, Piscataway, NJ, USA, 2nd edition, 1991.

[9] J. D. Kraus and R. J. Marhefka, Antennas for All Applications, chapter 16, 17, McGraw Hill, New York, NY, USA, 3rd edition, 2002.

[10] D. Sievenpiper, L. Zhang, R. F. Jimenez Broas, N. G. Alexöpolous, and E. Yablonovitch, "High-impedance electromagnetic surfaces with a forbidden frequency band," IEEE Transactions on Microwave Theory and Techniques, vol. 47, no. 11, pp. 2059-2074, 1999.

[11] P. S. Kildal, "Artificially soft and hard surfaces in electromagnetics," IEEE Transactions on Antennas and Propagation, vol. 38, no. 10, pp. 1537-1544, 1990. 

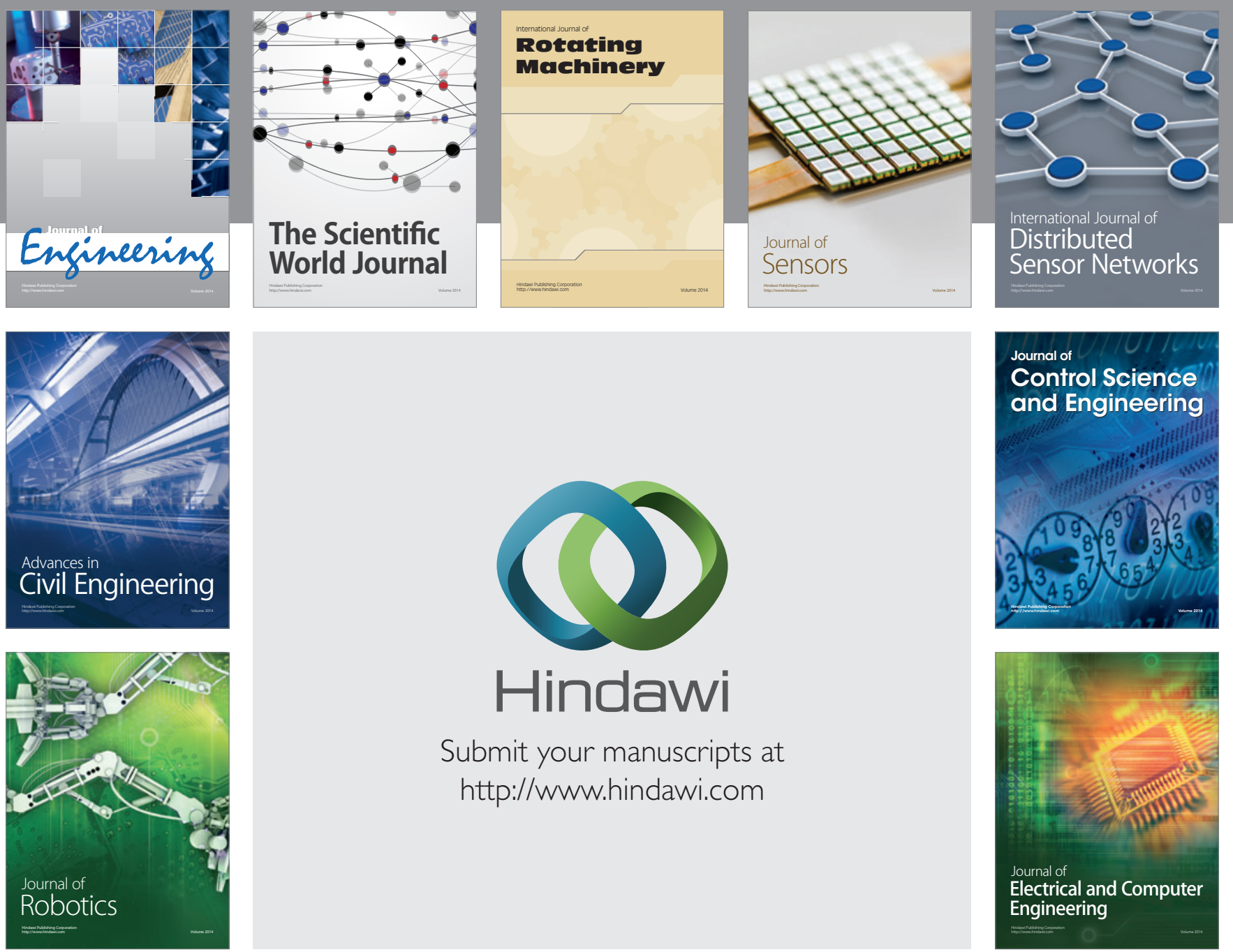

Submit your manuscripts at

http://www.hindawi.com
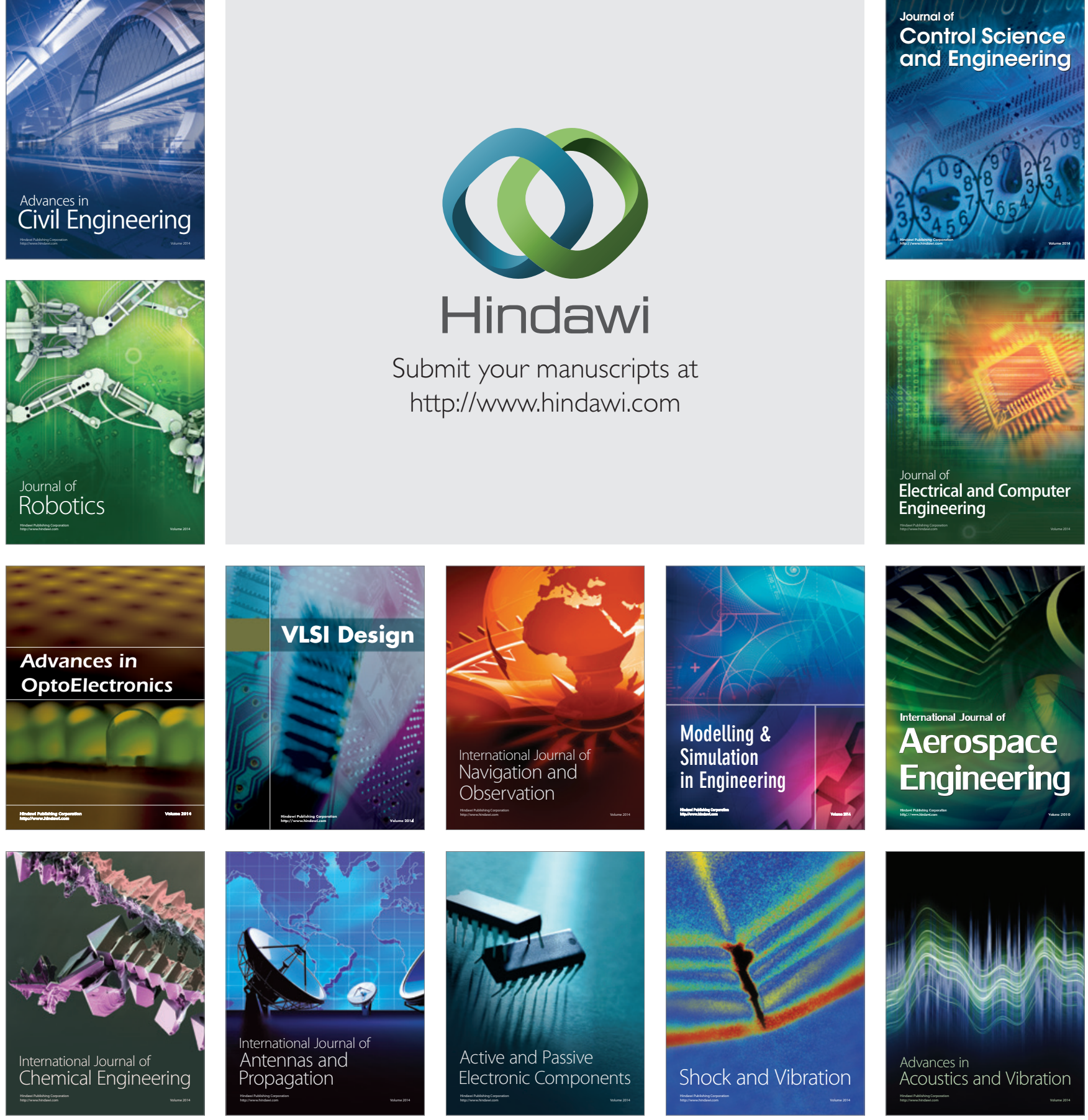